\title{
Bacteriological Profile and Antibiotic Susceptibility Pattern of Isolates of Wound Infection In Children Visiting Kanti Children Hospital
}

\author{
Gita Ghimire ${ }^{1}$, Ramnandan Prasad Chaudhary ${ }^{2}$, Binod Lekhak ${ }^{1}$ \\ ${ }^{1}$ Department of Microbiology, GoldenGate International College, TU \\ ${ }^{2}$ Department of Pediatrics, Kanti children hospital
} Corresponding author: Gita Ghimire, Department of Microbiology, GoldenGate International College, affiliated
to Tribhuvan University, Email: gghimire2011@yahoo.com,

\begin{abstract}
Objectives: The objectives of this study was to isolate and identify the bacterial etiological agent of wound infection and explore the status of methicillin-resistant Staphylococcus aureus ( MRSA), multidrug Resistant (MDR) and extended spectrum $\beta$-lactamase (ESBL) producers' strains in clinical specimens and to find the antibiotic susceptibility pattern.
\end{abstract}

Methods: A prospective cross sectional study design was conducted from period of February 2014 to October 2014 at Kanti Children Hospital, Kathmandu. The organisms were isolated and identified from pus sample by standard microbiological methods. Antimicrobial susceptibility test was performed by modified the Kirby Bauer disc diffusion method to evaluate the status of MRSA and MDR. ESBL detection was performed by the combined disc diffusion method.

Results: Out of 365 specimens collected between the age group below < 2 to 15 years, 210 (57.73\%) samples from male patients and 155 (42.47\%) from female patients. In the total samples processed, Gram-positive organisms were found to be more prevalent in which Staphylococcus aureus accounts for 135(47.20\%), followed by P. aeruginosa 62 (21.67\%), E. coli 29 (10.20\%), K. pneumoniae 27 (9.44\%), Acinetobacter spp. 20 (6.70\%), P. vulgaris 7 (2.44\%) and CoNS $6(2.10 \%)$. Among the S. aureus isolates, $29(21.48 \%)$ were found MRSA. Of the total Gram-negative organisms isolated, 74 (51.03\%) were MDR and $14(100 \%)$ ESBL producer, $(\mathrm{P}<0.01)$. S. aureus was found to be the most important and leading cause of wound infection in this study.

Conclusion: Thus, routine antibiotic susceptibility testing is recommended for empirical drug therapy and proper management of disease.

Key words: Bacteriological profile, MRSA, MDR, ESBL, wound infection.

\section{INTRODUCTION}

Wound infection is becoming a serious burden in surgical procedure hospitals. The prevalence of wound infections has been reported to be $40 \%$ in developing countries. The emergence of resistant strains has increased the morbidity and mortality associated with wound infections (Goswami et al. 2011; Kassam et al. 2017)

Wounds break the continuity of the skin and allow the organism to gain access to the tissues. The outcome of wound infection depends on the interaction of complex host and microbial factors (Shittu et al. 2003). According

Date of Submission: August 28, 2020

Published Online: December, 2020 to Bryan (1983), a wound can be considered infected if purulent materials drain from it even if a culture is found negative.

The emergence of resistant strains has increased the morbidity and mortality associated with wound infections (Goswami et al. 2011). The predominant isolates of wound bacteria are multidrug-resistant nosocomial pathogens such as methicillin-resistant Staphylococcus aureus (MRSA), Pseudomonas aeruginosa, Acinetobacter baumannii and enteric bacteria such as extended spectrum $\beta$-lactamase (ESBL) producing E. coli and Klebsiella spp. Of the various research

Date of Acceptance: October 29, 2020

DOI: https:/ / doi.org/10.3126/tujm.v7i0.33855 
conducted, the most prevalent organism involved in wound infections are S. aureus, Pseudomonas spp, E. coli. (Zafar et al. 2008).

The previous studied stated that the incidence of postoperative wound infection in children varies significantly, from 1.6 to $18.7 \%$, and even up to $27 \%$ for contaminated operations and up to $30 \%-40 \%$ for dirty-infected operations (Varik et al. 2011). The risk of surgical wound infection is based on the susceptibility of a surgical wound to microbial contamination (Raahave et al. 1986). The rate of surgical wound infection was found to be $4 \%$ in Tribhuwan University Teaching Hospital, Nepal (Banjara et al. 2002). Similarly, in case of post-operative cesarean wound infection rate in Patan Hospital was found to be $2.6 \%$ (Pandit et al. 2002). S. aureus is responsible for large variety of infections and predominates in surgical wound infections with prevalence rate ranging from $4.6 \%$ - 54.4\% (Batabyal et al. 2012). Overall prevalence of MRSA in clinical samples of hospitals located in Kathmandu Valley, Nepal was 62\% (Mukhiya et al. 2013).

This study focused to find the pattern of bacterial isolates from infected wounds and their antimicrobial susceptibility pattern. Therefore, the acquired data of the causative agents of infected wounds from this study would be helpful in the selection of empiric antimicrobial therapy and infection control measures. The other objective of this study was to isolate bacteria from infected wounds from different wards of the hospital.

Hence, this study gives the comprehensible image of current scenario of wound infection and their causative microorganisms in the study site. Moreover, antibiotic susceptibility testing will help understand the current susceptibility pattern of isolates toward the commonly used antibiotics and the corrects choice of antibiotics with respect to causative agents.

\section{MATERIALS AND METHODS}

Study design: A Prospective hospital based cross sectional study design was used.

Sample size: A total of 365 pus sample were collected from clinically defined children being affected by the wound infection.

Sampling methodology: Stratified a random sampling method was used for collecting data and pus sample.

Population: Populations for the study were patients visiting Kanti Children Hospital. The target patients were younger children up to 15 years of age with clinically diagnosed bacterial wound infection.

Ethical consent: Ethical approval was obtained from the Institutional Review Committee (IRC), Kanti Children Hospital, Maharajgunj, Kathmandu. After briefly informing the participant about the objectives of the study, verbal and written consent were also taken from all participants.

Sample collection: Wound samples were collected using sterile cotton swabs (fresh pus) but small screwcapped bottle a firmly stopper tube or syringe or a sealed capillary tube, and the patients name, age and gender were clearly written (Koneman et al. 2005). The sample was taken to the laboratory for further analysis without any delay. In case of delay, the samples were refrigerated at $4^{\circ} \mathrm{C}$.

Macroscopic examination: Macroscopic examination of the collected pus samples was conducted by observing its color and appearance and reported accordingly. However, data of macroscopic examination were not included in this study.

Culture: The samples were inoculated on Blood agar and MacConkey agar and incubated aerobically at $37^{\circ} \mathrm{C}$ for $24 \mathrm{hrs}$. Positive growth was identified by Gram staining, colony characteristics, hemolytic pattern in BA and standard biochemical tests. (Koneman et al. 2005; Cheesbrough 2000).

Identification of isolates: The isolated colony from plates showing positive growth was further preceded for identification. Plate showing no growth, mixed growth and bacterial growth of insignificant number was excluded from the study. The isolates were identified by standard microbiological methods as described by Collee et al. (1999). A single distinct colony from BA, MA for both Gram-negative and Gram-positive bacteria was picked up and inoculated on NA. It was incubated at $37^{\circ} \mathrm{C}$ for $24 \mathrm{hrs}$. After overnight incubation, the culture was used to perform biochemical tests and antibiotic susceptibility test.

Antibiotics susceptibility test: Antimicrobial susceptibility testing of the pus isolates were done by modified the Kirby-Bauer M2-A9 disk diffusion method as recommended by the Clinical and Laboratory Standards Institute (CLSI 2014) using Mueller Hinton agar (MHA). For Staphylococcus MHA incorporated 
with $0.2 \% \mathrm{NaCl}$ was used.

Screening of Methicillin-Resistant S. aureus (MRSA): Screening of MRSA was based on the disc diffusion method using cefoxitin $(30 \mathrm{mcg})$. The diameter of the zone of inhibition of $\leq 21 \mathrm{~mm}$ was considered as methicillin resistant (MRSA), whereas diameter of the zone of inhibition $\geq 22 \mathrm{~mm}$ was considered as methicillin susceptible (MSSA) (CDC 2014).

Screening of MDR: The organisms showing resistant to more than three classes of antibiotics were taken as Multi-drug resistant isolates (Magiorakos et al. 2010 and CDC 2006). In this study, four classes of antibiotics were chosen.

\section{Detection of ESBLs}

Screening of ESBL producers: Bacterial isolates were first tested with at least one of the five antibiotics i.e., Ceftriaxone $(30 \mu \mathrm{g})$ according to the CLSI screening criteria. The isolates were suspected to produce ESBL if the zone of inhibition was $\leq 25 \mathrm{~mm}$. Ceftriaxone (30 $\mu \mathrm{g}$ ) was included in the initial line of AST for screening.

\section{Confirmation of ESBLs}

The phenotypic combination disc method: The suspected ESBL isolates were tested for confirmation by using the Combination Disc Method, using Cefotaxime $(10 \mu \mathrm{g})$ and cefotaxime $(10 \mu \mathrm{g})$ plus clavulanate $(1 \mu \mathrm{g})$, Cefotaxime $(30 \mu \mathrm{g})$ and Cefotaxime $(30 \mu \mathrm{g})$ plus clavulanate $(10 \mu \mathrm{g})$ and ceftazidime $(30 \mu \mathrm{g})$ and ceftazidime $(30 \mu \mathrm{g})$ plus clavulanate $(10 \mu \mathrm{g})$. An increase in zone diameter of $\geq 5 \mathrm{~mm}$ in the presence of clavulanate from any or all of the set was confirmed as ESBL producers.

Quality control: The quality of each agar plate prepared was maintained by incubating one plate of each batch in the incubator. Control strains of E. coli ATCC 25922 and S. aureus 25923 were used for the identification test and for the standardization of Kirby-Bauer test and for correct interpretation of the diameter of inhibition zones. The quality of sensitivity test was maintained by maintaining the thickness of MHA to $4 \mathrm{~mm}$ and the $\mathrm{pH}$ of 7.2-7.4.

Purity plate for each biochemical test was maintained to ensure pure culture, inocula used and to assess that the biochemical tests were undertaken in aseptic conditions.

Statistical analysis: All the data obtained were statistically analyzed using Statistical Package for Social Science (SPSS) version 17 software packages. The chi-square, one way ANOVA was used according to should determine a significant association between different factors for the causation of wound infections.

\section{RESULTS}

Out of 365 pus sample collected and processed, where 38 (10.42\%) samples were from surgical wound infection patients, 115 (31.50\%) from burn ward and $212(58.08 \%)$ from OPD.

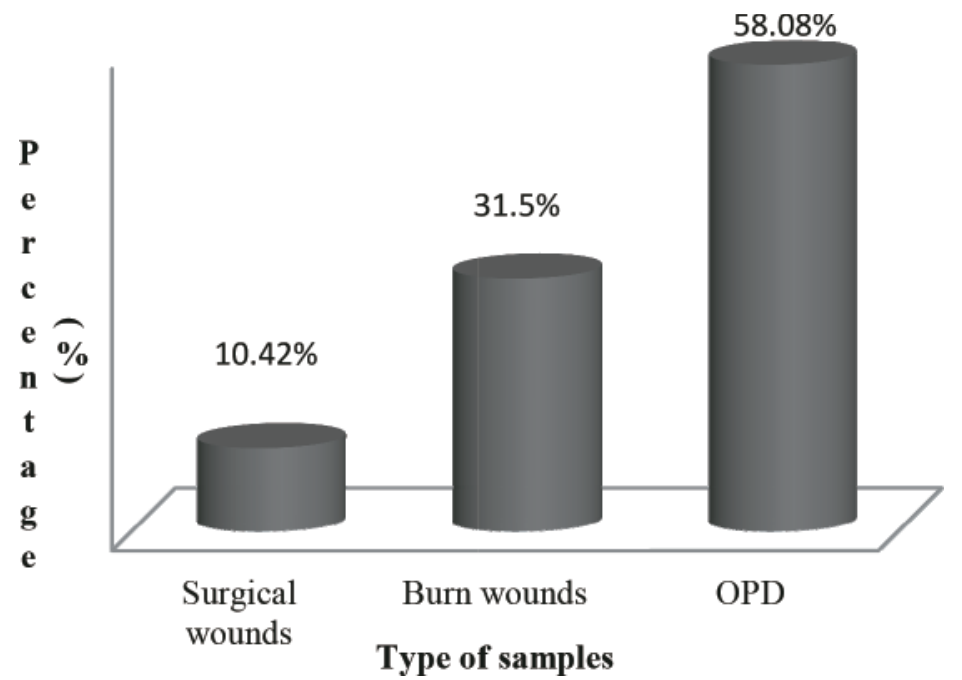

Figure 1: Types and distributions of Samples 
Among the total pus sample, 248 (67.95\%) were collected by pus aspirates and 117 (32.05\%) pus by swabs. Among the collected pus samples, 210 (57.73\%) were from male patients and 155 (42.47\%) from female patients.

The maximum 144 (39.45\%) pus samples were collected from patients of the age group of $2-5 y e a r s, ~(m a l e=76$ and female $=68$ ), followed by the age group $<2$ years with $136(37.26 \%)$ samples $($ male $=84$ and female $=52)$ and the least samples were 15 (4.11) from the age group of more than 10 .
Among the 365 samples collected, 286 (78.25\%) samples showed growth and $94(21.64 \%)$ did not detect any growth. Similarly, Out of 286 isolates, Gramnegative bacteria were predominating constituting 145 (50.70\%) and Gram-positive bacteria constituted 141 (49.30\%). Among total bacterial isolates, $S$ aureus was predominant species with 135 (47.20\%), followed by $P$. aeruginosa $62(21.67 \%)$, E. coli $29(10.20 \%)$, K. pneumoniae $27(9.44 \%)$, Acinetobacter spp. $20(6.70 \%)$, P. vulgaris 7 (2.44\%) and CONS 6 (2.10\%) in number.

Table 1: Types of bacterial isolates in wound specimens

\begin{tabular}{ccc}
\hline S.N. & Name of Organisms & No. (\%) \\
\hline 1 & Acinetobacter spp. & $20(6.70)$ \\
2 & Escherichia coli & $29(10.20)$ \\
3 & Klebsiella pneumoniae & $27(9.44)$ \\
4 & Proteus vulgaris & $7(2.44)$ \\
5 & Pseudomonas aeruginosa & $62(21.67)$ \\
6 & CoNS & $6(2.10)$ \\
7 & Staphylococcus aureus & $135(47.20)$ \\
\hline
\end{tabular}

Among 145 Gram-negative bacteria, P. aeruginosa

$27(18.62 \%)$, E. coli 29 (13.79), Acinetobacter spp. 20 accounted for $62(42.76 \%)$ followed by K. pneumoniae

$(13.79 \%)$, P. vulgaris 7 (4.83\%).

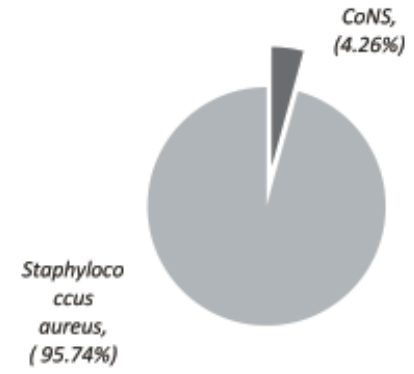

Figure 2: Types of Gram-positive bacteria isolates in wound specimens

Similarly, among 141 Gram-positive bacteria isolates,

(95.74\%) followed by CONS i.e. $6(4.26 \%)$.

S. aureus was present in the highest number i.e. 135

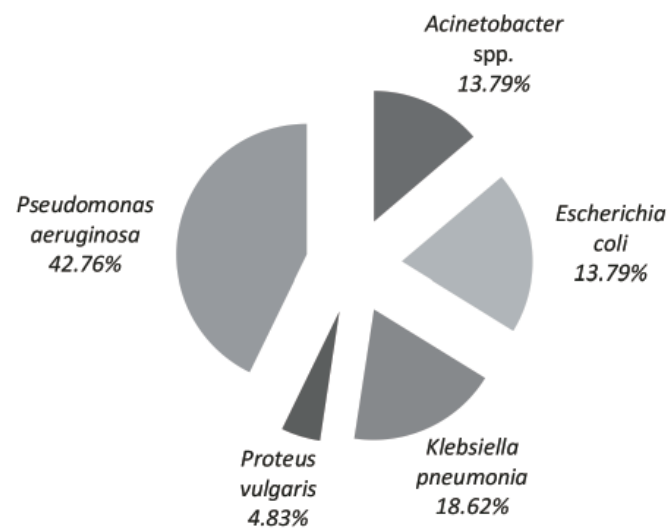

Figure 3: Types of Gram negative bacterial isolates in wound specimens 


\section{Antibiotic sensitivity pattern of Isolates}

Piperacillin and Tazobactam were the most effective drug showing $85 \%$ sensitivity followed by Chloramphenicol (75\%) whereas Cotrimoxazole, Amoxiclave was less effective against Acinetobacter spp.

Similarly, ciprofloxacin, Gentamycin and amoxicillin and Chloramphenicol were the most effective drug of choice and Penicillin was the least effective toward CONS isolates.

However, Cefixime, Nitrofurantoin, Amoxiclave and Nalidixic acid was the most effective for E. coli and least was Amikacin.

Nalidixic acids, Cefixime, Norfloxacin were effective antibiotics and Amoxiclave was least effective antibiotics of choice for K. pneumoniae.

Similarly, Chloramphenicol (100\%), Piperacillin (71.43\%), Ciprofloxacin (71.43\%), Nitrofurantoin
(71.43\%) were the most effective antibiotics for Proteus vulgaris and Norfloxacin was least effective. The sensitivity pattern was statistically found significant with $\mathrm{p}<0.01$.

Piperacillin and Tazobactam (82.26\%), Clindamycin $(87.10 \%)$, Amikacin and Imipenem $(76.74 \%)$ was the most effective antibiotics, whereas, Ceftazidime and

Gentamycin was least effective for $P$. aeruginosa. The sensitivity pattern was statistically found significant with $\mathrm{p}<0.001$.

Likewise, Cotrimoxazole (83.70\%), Amikacin (74.81\%) was the most effective antibiotics and Penicillin (20\%) was least effective antibiotics for S. aureus $(\mathrm{p}<0.001)$.

\section{MRSA DISTRIBUTIONS}

Among S. aureus 135(47.20\%) isolates, 106 (78.52\%) were Methicillin-Sensitive S. aureus (MSSA) and 29 (21.48\%) were Methicillin-Resistant S. aureus (MRSA).

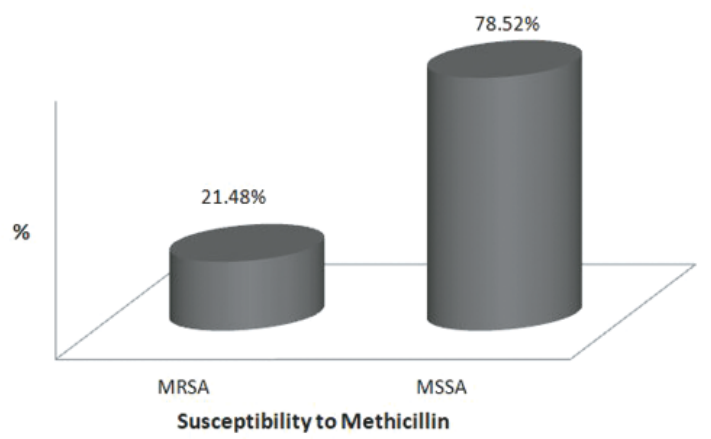

Figure 4: Distribution of MRSA and MSSA among S. aureus isolates

\section{Distribution of MRSA based on the Age group,} Gender, patient type and department:

The highest rate of infection by MRSA isolates was found to be $13(44.8 \%)$ in the age group 2-5 years, followed by age group $<2$ years $9(31.08 \%), 10-15$ years $6(20.69 \%), 5-10$ years $1(3.45 \%)$ respectively. The rate of MRSA infection was found to be highest among pediatrics than in adults.

Similarly, the rate of infection due to MRSA was higher in males 22 (65.52\%) than in females 10 (34.48\%).

Moreover, inpatient 17 (58.62\%) were more susceptible by MRSA than outpatient 65 (61.32\%)

Similarly, among the 29 (21.48\%) MRSA isolates, the highest MRSA isolates were from 16 (55.17\%) OPD and lowest isolates were 6 (20.69\%) from Surgical ward.

\section{MDR DISTRIBUTION}

The distribution of MDR among Gram-negative isolates: Among the 286 culture positive isolates, 145 were Gram-negative organisms. In total Gram-negative isolates, 74 (51.03\%) were multidrug resistant (MDR).

MDR isolates were found to be most prevalent among the age group below 1 years $30(40.45 \%)$, followed by age group 2-5 years 27 (36.49\%), 6-10 years 16 (21.61\%), 11-15years $1(1.35 \%)$, respectively.

Similarly, Gram-negative MDR isolates were found to be most prevalent in females 32(43.24\%) than in male patients $42(56.76 \%)$.

Likewise, the distribution of MDR was high in the Outpatient 32 (43.24\%) than Inpatient 42 (56.76\%). 
Among 74 MDR isolates, the highest distribution was found to be from OPD 37 (50.00\%), followed by burn ward $30(40.54 \%)$ and least from surgical ward 7(9.46\%).

Out of 145 gram negatives isolates, MDR isolates accounted for 74 (51.03\%) in which a higher number of Pseudomonas aeruginosa isolates, 23 (31.08\%) were multidrug resistant, followed by Escherichia coli 22 (29.73\%) and K. pneumoniae 18 (24.32\%), Acinetobacter spp. $(9.46 \%)$ and Proteus vulgaris $4(5.41 \%)$

\section{Distributions of ESBL Producers}

Among the isolates, 72 were ESBL suspected, in which14 $(19.44 \%)$ were found to be ESBL positive and $58(80.56 \%)$ were found to be negative by confirmatory tests. In confirmation test, two combination

Disks were used, namely the ceftazidime clavulanate and Cefotaxime-clavulanate Among the 14 ESBL positive isolates, most predominant was found to be E. coli with 8/19 (42.11\%) followed by K. Pneumonia, P. aeruginosa and Proteus vulgaris $(\mathrm{P}<0.01)$.

Table 2: ESBL production from various isolates

\begin{tabular}{lccccc}
\hline \multirow{2}{*}{ Organism } & No. Of ESBL & \multicolumn{5}{c}{ ESBL confirmatory Test } \\
\cline { 3 - 6 } & suspected & Positive & $\%$ & Negative & $\%$ \\
\hline Acenetobacter spp. & 7 & 1 & 14.29 & 6 & 85.71 \\
E. coli & 19 & 8 & 42.11 & 11 & 57.89 \\
Proteus vulgaris & 2 & 0 & 0 & 2 & 100 \\
K. pneumoniae & 14 & 3 & 21.43 & 11 & 78.57 \\
P. aeruginosa & 30 & 2 & 6.67 & 28 & 93.33 \\
\hline Total & $\mathbf{7 2}$ & $\mathbf{1 4}$ & $\mathbf{1 9 . 4 4}$ & $\mathbf{5 8}$ & $\mathbf{8 0 . 5 6}$ \\
\hline
\end{tabular}

Table 3: Pattern of ESBL production using 2 combination disks

\begin{tabular}{|c|c|c|c|c|c|}
\hline $\begin{array}{c}\text { Combination disk } \\
\text { used }\end{array}$ & Criteria for confirmation & $\begin{array}{l}\text { No. of suspected } \\
\text { ESBL producer }\end{array}$ & $\begin{array}{l}\text { No. of confirmed } \\
\text { cases }\end{array}$ & $\begin{array}{l}\text { Total confirmed } \\
\text { cases }\end{array}$ & $\begin{array}{l}\text { Negative after } \\
\text { confirmation }\end{array}$ \\
\hline CTX: CTX+CV & Increase in zone size of $\geq$ & & 12 & & \\
\hline $\mathrm{CAZ}, \mathrm{CAZ}+\mathrm{CV}$ & $\begin{aligned} 5 \mathrm{~mm} \text { with } & \geq 1 \text { combination } \\
& \text { disks }\end{aligned}$ & 72 & 14 & 14 & 58 \\
\hline
\end{tabular}

Note: CPD-Cefpodoxime, CAZ-Ceftazidime, CTX-Cefotaxime, CV-Clavulanate

\section{DISCUSSION}

In this study, out of 365 wound samples collected, 286 $(78.25 \%)$ were found to be culture positive and only 94 $(21.64 \%)$ showed no growth.

This study is comparable to the study by Nepal et al. (2008) and Shrestha (2009) in which $80.6 \%$ and $72.4 \%$ showed bacterial growth. This might be due to similar culture medium used and growth environment condition for isolation of organisms.

In this study, the higher number of growth was observed in in-patients $17(58.62 \%)$ than in outpatients. This may be due to weak in immune status after wound infection and acquirement of nosocomial infection facilitates the wound infection. Similar results reported by Mahat et al. (2017) the higher number of growth positive cases in in-patients

The occurrence of higher number of male patients $57.73 \%$ than female patients from the collected of pus samples may be due to the involvement of higher outdoor activities like playing as compared to females and more chances of getting accidents during outdoor activities. This finding was supported by similar type of study conducted by Mahat et al. (2017) and KC et al. (2013).

In this study, Gram-negative rods (50.70\%) were the predominant and leading cause of wound infections. In similar study conducted by Yakha et al. (2014) and Mahat et al. (2017) Gram negative bacteria were found predominant. Gram negative bacteria isolation was higher, as they are more common aerobes and facultative anaerobes in abscesses and skin wound. Gram negative bacteria increases in the cases of Hospital acquired infections (HAIs). This might be due to high resistances to antibiotics showed by Gram negative bacteria compared to Gram-positive isolates, and therefore their persistence in infected wounds. Furthermore, chronic wounds were infected by multiple Gram-negative rods. The multiple bacterial infections in this case might be due to impaired immune responses. But Maharjan et al. (2020) Khanam et al. (2018) and Pandey et al. (2017) showed high isolation rate of Gram positive bacteria. 
In this study, S. aureus accounted for 135 (47.20\%) followed by P. aeruginosa $62(21.67 \%)$, E. coli $29(10.20 \%)$, K. pneumoniae 27 (9.44\%), Acinetobacter spp. 20 (6.70\%), P. vulgaris $7(2.44 \%)$ and CONS $6(2.10 \%)$.

S. aureus was the leading isolates of wound infection. High rate of isolation may be due to its presence of normal flora nasal cavity and skin of the individuals and unhygienic behavior like contact of wound site with the hand contaminated with the nasal discharge may be the possible reason. Similar type of results was found by Maharjan et al. (2020). Nazeer et al. (2014) found similar results in which the most predominant isolate from a wound infection was Staphylococcus aureus (37.12\%) followed by Klebsiella species (20.45\%).

The study reported by Karkee (2008) also supported the finding of this study in which the most common bacterial isolates $(46.58 \%)$ were S. aureus. E. coli (12.38\%) emerged as the next common organism causing wound infection followed by CONS $(11.40 \%)$ and $P$. aeruginosa $(7.49 \%)$. The least common bacteria isolated were C. freundii $(0.65 \%)$. In Saudi Arabia, Abussaud (1996) isolated S. aureus (35\%), P. aeruginosa (25\%) and Klebsiella spp. (10\%) as the major causative agents.

However, different studies have shown $P$. aeruginosa as the leading cause of burn wound infections. A study by Mousa (1997) to assess the rate of burn wound infection by aerobic bacteria and found that 19.1\% of the wound infection was caused by P. aeruginosa. Similar studies on burn wound infection by Nasser et al. (2003) showed that P. aeruginosa (21.6\%) as the most common isolate. This result supports the findings of this study in which the prevalence rate of $P$. aeruginosa was found to be $62(21.67 \%)$. In contrast to this finding, studies carried out by Ranjan et al. (2011) and Mahat et al. (2017) also showed Pseudomonas spp. was the most prevalent bacteria among the total cases with $27.8 \%$ and $29.6 \%$ and $34.55 \%$ respectively. This might be due to chances of nosocomial infection by $S$. aueus and $P$. aeroginosa both in in-patients and out-patients

In a study by Shah et al. (1997) at TU Teaching Hospital, $11.43 \%$ of CONS was reported from pus sample, which was far-away to this finding. Shampa et al. (2006), in their study to determine the prevalence of $P$. aeruginosa and its antimicrobial sensitivity pattern found $32 \%$ prevalence rate of $P$. aeruginosa of all pathogens isolated. This study agreed with the study done in Africa by Oguntibeja et al. (2004) where 33\% of the isolates were $P$. aeruginosa in post-operative wound infections. Similar results were found in the study by Anbumani et al. (2006). Giacometti et al. (2000) studied microbiology of wound infection in Italy and found that the prevalence of $S$. aureus $(28.2 \%), P$. aeruginosa $(25.2 \%)$, E. coli $(7.8 \%)$, which gave a different result than our study.

Cotrimoxazole, Amikacin, Ciprofloxacin, Gentamycin, Amoxycillin were effective drug of choice for Grampositive. In contrast to this study, Shrestha (2009) found Chloramphenicol as the most effective drug with susceptibility $(85.58 \%)$ followed by ceftriaxone (77.88\%), Amikacin (72.12\%) and Ofloxacin (67.13\%) against Gram-positive isolates least was found Cloxacillin (20.19\%) and Cotrimoxazole (40.38\%). In this study, Chloramphenicol, Piperacillin, Nitrofurantoin, Amoxiclave and Nalidixic acid were found to be the most effective drug for treatment for Gram-negative isolates. In contrast to this study, Shrestha (2009) reported that against Gram-negative isolates from a wound infection, Amikacin was found to be the most effective drug (55.84\%) followed by Ofloxacin (51.95\%), Ciprofloxacin $(45.45 \%)$ and Ceftriaxone $(40.26 \%)$ and the least effective was found to be Cloxacillin (16.88\%). Similarly, sensitivity pattern of Gram-negative bacteria to Amikacin observed in this study was similar to study by Agnihotri et al. (2004), in which Amikacin was found to be the most effective antibiotic for Gramnegative bacteria.

In this study, among 286 culture positive isolates, $135(47.20 \%)$ were S. aureus and $29(21.48 \%)$ were Methicillin-Resistant $S$. aureus (MRSA). This finding in this study was supported by research conducted by Kshetry (2014) in which of the 149 culture positive isolates, $83(55.7 \%)$ were S. aureus. Out of total S. aureus isolates, $27(32.5 \%)$ were MRSA.

Among 286 culture positive isolates, 145 were Gramnegative organisms in which 74 (51.03\%) were multidrug resistant (MDR). The rate of MDR was found to be higher $30(40.45 \%)$ in the age group $<2$ years. Higher number 23 (31.08\%) of MDR was found to be Pseudomonas aeruginosa followed by Escherichia coli $22(29.73 \%)$ and K. pneumoniae 18 (24.32\%), Acinetobacter spp. $(9.46 \%)$ and Proteus vulgaris 4(5.41\%). Outcome of prevalence of MDR depends on various factors, MDR criterion being the chief one followed by the types of antibiotics used in antibiogram and study population. 
Bhandari (2016) reported similar results in which 138 isolates were evaluated, MDR isolates accounted for $100 / 138(72.45 \%)$. A relatively lower prevalence of MDR, the prevalence of $56.09 \%$ and $35.2 \%$ were found in the study by Tuladhar et al. (2003), whereas $80 \%$ MDR has been reported in the study by Menon et al. (2006) and 81.1\% MDR has been reported in the study carried by Waikhan and Devis (2012). Similarly, in contrast to this study, Bhandari (2014) Pokhrel et al. (2006) reported that the high number of E. coli isolates 64 (72.7\%) as multidrug resistant followed by Pseudomonas aeruginosa 11 (91.7\%) and K. pneumoniae 6(75\%). Outcome of prevalence of MDR depends on various factors, MDR criterion being the chief one followed by the types of antibiotics used in antibiogram and study population. The emergence of MDR is clearly related to the quantity of antibiotics and how they are being used (Levy, 1991).

Among the 14 ESBL positive isolates, the majority consist of $E$. coli with 8/19 (42.11\%) followed by $K$. Pneumonia, P. aeruginosa and Proteus vulgaris. Of the total primary screened E. coli with 8/19 (42.11\%) were ESBL positive.

This study was supported by Bhandari (2016), Out of 68 screened positive samples, $44(64.7 \%)$ were found to be ESBL positive isolates. A similar result was obtained in the study by Poudyal (2010), which showed 62.7\% positive after confirmation test. E. coli $34 / 44$ (77.3\%) was found to be most predominant ESBL positive isolates followed by K. pneumoniae, Pseudomonas aeruginosa and Enterobacter spp each with 2/44 (4.5\%) isolates. The pattern of E. coli to be the most predominant ESBL positive isolates was found in various other studies conducted by Poudyal (2010) and Baral (2011).

We observed 14 (19.44\%) of ESBL among total 72 isolates. A previous study by Ashrafian et al. (2012), Srisangkaew, and Vorachit (2003) found 32.7\% and $40 \%$ ESBL, respectively, whereas various other similar studies such as research performed by Singh (2013) observed 49\% ESBL producers, Rijal (2010) observed 28.88\% and Pokhrel et al. (2006) observed 16\% ESBL producers; however, the global prevalence of ESBL producing organisms presently varies from $<1 \%-74 \%$ (Thokar et al. 2010). The prevalence of ESBLs among clinical isolates varies from country to country and from institution to institution. These differences may be due to geographical variations, local antibiotic prescribing habits (Pokhrel et al. 2006).

\section{CONCLUSIONS}

S. aureus was the most important and leading cause of wound infection that was the organism of emphasis of this study. Antibiotic susceptibility testing of all isolates was performed. Antibiotic susceptibility testing of all isolates showed that Piperacillin, Nitrofurantoin, Amoxyclav and Nalidixic acid, imipenem was the most effective drugs for Gram-negative bacteria and vancomycin, Cotrimoxazole, Amikacin, Ciprofloxacin, Gentamycin, Amoxycillin and chloramphenicol was the most effective drug for Gram-positive organisms. In case of Gram-negative isolates MDR pattern observed which accounts for 74/145 (51.03\%). Similarly, ESBL was primarily screened by Ceftriaxone/Ceftazidime (CAZ) and confirmation by the combined disk method, confirmed ESBL producers were found to be 14 (19.44\%). E. coli is leading ESBL Isolates. Thus, routine antibiotic susceptibility testing is recommended for empirical drug therapy.

\section{CONFLICT OF INTEREST}

The authors declare no conflict of interest.

\section{REFERENCES}

Abbussaud MJ (1996) Incidence of wound infection in three different departments and the antibiotic sensitivity pattern of the isolates in a Saudi Arabian hospital. Acta Microbiol Immunol Hunga 43: 301-305.

Anbumani N, Kalyan J and Mallika M (2006) Epidemiology and microbiology of wound infections. Ind J Pract Doct 3: 182-205.

Agnihotri N, Gupta V and Joshi R (2004) Aerobic bacterial isolates from burn wound infections and their antibiograms - a five-year study. Burns 30(3): 241-243.

Ashrafian F, Askari E, Kalamatizade E, Ghabouli Shahroodi MJ and Naderi-Nasab M (2012) The Frequency of Extended Spectrum Beta Lactamase (ESBL) in Escherichia coli and Klebsiella pneumoniae. J Med Bacteriol 1(3): 12-19.

Banjara MR, Sharma AP, Joshi AB, Tuladhar NR, Ghimire P and Bhatta DR (2002) Surgical Wound Infections in Patients of Tribhuvan University Teaching Hospital. Surgical Wound Infections 1: 41-45. 
Baral R, Khanal B and Acharya A (2011) Antimicrobial susceptibility patterns of clinical isolates of Staphylococcus aureus in Eastern Nepal. Health Renaissance 9: 78-82.

Batabyal B, Biswas S, and Mandal B (2012) Isolation of Imipenem Resistant Staphylococcus aureus from Post-Operative Pus Sample in Oral and Maxillofacial infections. Res J of Pharm Biol and Chem Sc.

Bhandari, R., Pant, N.D., Poudel, A. Sharma M (2016) Assessment of the effectiveness of three different cephalosporin/clavulanate combinations for the phenotypic confirmation of extended-spectrum beta-lactamase producing bacteria isolated from urine samples at National Public Health Laboratory, Kathmandu, Nepal. BMC Res Notes 9: 1-5.

Bryan CS, Dew CE, Reynolds KL (1983) Bacteremia associated with decubitus ulcers. Arch Intern Med 143(11): 2093-95.

Centres for Diseases Control (CDC) (2014) Methicillin Resistant Staphylococcus aureus. URL: https:// www.cdc.gov/mrsa/lab/index.html

Cheesbrough M (2000) District laboratory practice in tropical countries. Cambridge University Press, London 2: 225-392.

CLSI (2013) Performance standards for antimicrobial susceptibility testing; twenty-second Informational supplement, 33. CLSI Document M100-S20. Wayne, PA.

Clinical and Laboratory Standards Institute/NCCLS (2014) Performance Standards for antimicrobial susceptibility testing; 15th informational supplement. CLSI/NCCLS M100-S15. Clinical and Laboratory Standards Institute.

Collee JG, Fraser AG, Marmion BP and Simmons A (1999) Mackie and McCartney's practical medical microbiology. $14^{\text {th }}$ edition, Churchill Livingstone pp. 26-196.

Kassam NA, Damian DJ, Kajeguka D, Nyombi B and Kibiki GS (2017) Spectrum and antibiogram of bacteria isolated from patients presenting with infected wounds in a Tertiary Hospital, northern Tanzania. BMC Res Notes 10:1-6.

Kshetry AO (2014) Antibiogram of bacteria isolated from wound exudates. M. Sc. Dissertation Submitted to the Department of Microbiology GoldenGate International College, Tribhuvan. Pp. 5-10.

Giacometii A, Cirioni O, Schimizzi AM, Del Prete MS, Barchiessi F, D'Errico MM, Petrelli E and Scalise G (2000) Epidemiology and microbiology of surgical wound infections. J Clin Microbiol 38(2): 918-922.

Karkee P (2008) Bacterial Isolates and their Antibiogram from Wounds and Abscesses of Surgical Outpatients Visiting Bir Hospital. M. Sc. Dissertation Submitted to the Department of Microbiology, Tribhuvan Uinversity pp. 13-29.

KC R, Shrestha A, Sharma VK (2013) Bacteriology Study of Wound Infection and Antibiotic susceptibility Oattern of Isolates. Nepal J Sci Technol 14(2): 143150.

Khanam RA, Islam MR, Sharif A, Parveen R, Sharmin I, Yusuf MA (2018) Bacteriological profile of pus with antimicrobial sensitivity pattern at a Teaching hospital in Dhaka city. Bangladesh. J Infect Dis 5(1):10-14.

Koneman WK, Allen SD, Janda WM, Schreckenberger PC, Propcop GW, Woods and GL and Winn WC (2005) Jr. Philadelphia Color Atlas and Textbook of Diagnostic Microbiology, 6th ed. LippincottRaven Publisher: 624-662.

Levy SB (1991) Antibiotic availability and use: Consequences to men and his environment. J Clin Epidemial 44: 635-875.

Magiorakos AP, Srinivasan A, Carey RB, Carmeli Y, Falagas ME, Giske CG, Grundmann H, Harbarth S, Hindler JF, Kahlmeter G, Olsson-Liljequist B, Paterson DL, Rice LB, Suetens C, Stelling J, Struelens MJ, Vatopoulos A, Weber JT and Monnet DL (2010) Multidrug-resistant (MDR), extensively drug-resistant (XDR) and pandrug-1 resistant (PDR) bacteria in healthcare settings. Expert proposal for a 2 standardized international terminology.

Magiorakos AP, Srinivasan A, Carey R, Carmeli Y, Falagas M, Giske C, Harbarth S, Hindler J, Kahlmeter G and Olsson-Liljequist B (2012) Multidrug-resistant, extensively drug-resistant 
and pandrug-resistant bacteria: an international expert proposal for interim standard definitions for acquired resistance. Clin Microbiol and Infect 18(3): 268-281.

Maharjan N, Mahawal BS (2020) Bacteriological Profile of Wound Infection and Antibiotic Susceptibility Pattern of Various Isolates in a Tertiary Care Center. J Lumbini Med Coll 8(2):1-7.

Mahat P, Manandhar S, Baidya R (2017) Bacteriological Profile of Wound Infection and Antibiotic Susceptibility Pattern of the Isolates. J Microbiol $\operatorname{Exp}$ 4(5).

Mousa HA (1997) Aerobic, anaerobic and fungal burn wound infections. J Hosp Infect 37: 317-323.

Nasser S, Mabrouk A and Maher A (2003) Colonization of burn wounds in Ain Shams University Burn Unit. Burns 29: 229-233.

Nazeer HA, Shaik KM, Kolasani BP (2014) Aerobic bacteriology of wound infections with special reference to MRSA. J Clin Exp Res 2(1): 74-79.

Oguntibeju OO and Nwobu RA (2004) Occurrence of $P$. aeruginosa in post operative wound infection.Pak J Med Sc 20(3): 187-191.

Pandey U, Raut M, Bhattarai S, Bhatt PR, Dahal PR (2017) Bacteriological profile and antibiogram of bacterial isolates from pus sample in tertiary care hospital of Kathmandu. Tribhuvan Uni J Microbiol 4(1):55-62.

Poudyal S (2010) Prevalence of $\beta$-Lactamases producing multidrug resistant bacterial pathogens isolated from different clinical samples at National Public health laboratory, Nepal. M.Sc. Dissertation submitted to the Central Department of Microbiology, Tribhuvan University, Kathmandu, Nepal.

Pokhrel BM, Koirala J, Mishra SK, Dahal RK, Khadka P and Tuladhar NR (2006) Multidrug resistance and extended spectrum beta-lactamase producing strains causing lower respiratory tract and urinary tract infection. J Inst Med 8: 30-34.

Ranjan KP, Ranjan N, Bansal SK, Arora DR (2011)
Prevalence of Pseudomonas aeruginosa in Postoperative Wound Infection in a Referral Hospital in Haryana. India J Lab Physicians 2 (2): 74-77.

Rijal N (2010) Antibiogram of Carbapnems Toward Extended Spectrum Beta lactamase Producers. M.Sc. Dissertation submitted to the Central Department of Microbiology, Tribhuvan University, Kathmandu, Nepal. pp 5-46.

Shah PK, Pokharel BM and Sharma AP (1997) The incidence of coagulase negative staphylococci in Nepalese people. J Nep Med Assoc 123(36): 296-300

Shampa A, Bhattacharjee A, Garg A and Sen MR (2006) Antimicrobial susceptibility of $P$. aeruginosa isolated from wound infection. Ind J Dermatol 51(4): 286-288

Shrestha P (2009) Study on Bacteriological Profile of Infected Wound from Patients Visiting Kanti Children's Hospital, Maharajgunj, Kathmandu, Nepal. M. Sc. Dissertation Submitted to the Department of Microbiology, Tribhuvan. pp. 5-25.

Shittu AO, Oriade D and Oyedepo EAR (2003) Wound infections in two health institutions in Ile -Ife, Nigeria: Results of cohort study. Ostomy/Wound Management 49 (5): 52-57.

Tuladhar P (1999) A prospective study on bacteriology of wound infection at TUTH. A dissertation presented to the Central Department of Microbiology, Tribhuvan University, Kathmandu, Nepal.

Waikhom KD and Devi KS (2012) Emergence of multidrug resistant bacterial infection in $\mathrm{HIV} /$ AIDS cases. The Health 3(2): 49-52.

Yakha JK, Sharma AR, Dohal N, Lekhak B, Banjara MR (2014) Antibiotic Susceptibility Pattern of Bacterial Isolates Causing Wound Infection Among the Patients Visiting B \& B Hospital. Nepal J Sci and Technol 15(2): 91-96.

Zafar A, Anwar N and Ejaz H (2008) Bacteriology of Infected Wounds - A Study Conducted At Children's Hospital Lahore. Biomedica 24: 71-74. 